\title{
La "escrupulosa mezquindad" de la escritura en Dublineses, de James Joyce
}

\author{
Luz Aurora PIMENTEL \\ Universidad Nacional Autónoma de México
}

De acuerdo con la intención manifiesta de Joyce de escribir los cuentos de $D u$ blineses "en un estilo de escrupulosa mezquindad", este artículo aborda la colección tomando en cuenta el contexto histórico que explica el deterioro de Dublín - patente en las descripciones de la ciudad - a partir del Acta de la Unión de 1801 y el desastre humano que significó la gran hambruna de mediados del siglo XIX. En este contexto de depresión urbana y económica — agravada por la obsesión que tiene el irlandés de emigrar, "de huir de la "isla condenada que se moría de hambre' y encontrar un refugio en otra parte"- se analiza, en un primer momento, la temática general de los cuentos, para luego particularizar el análisis en algunos de ellos — "Las hermanas", "Una nubecilla" y "Los muertos". Destaca en el análisis no sólo la "escrupulosa mezquindad" del estilo, el cual podría considerarse como la marca del sobrio realismo de los cuentos, sino la vertiente simbólica que reside en lo no dicho, en la historia virtual, aquella que por la técnica narrativa de la indirección no ha sido contada, la historia ausente (gnomon) que proyecta su sombra o irradia su luz en la epifanía típicamente joyceana; todo aquello, en fin, que constituye la dimensión poética y simbólica de los relatos de Dublineses.

Palabras ClaVe: James Joyce, Dublineses.

Given Joyce's statement that he had written Dubliners "for the most part in a style of scrupulous meanness", this article explores the stories in the collection under this light and taking into account the historical context behind the urban depression apparent in the stories, from the 1801 Act of Union, through the Great Famine in the middle of the XIXth century. In this context of urban and economic depression aggravated by the inveterate obsession of the Irish "to escape, at all costs, from the 'doomed and starving island' and find safety elsewhere", the main themes of the collection are first revised in the light of this context; then, some of the stories are subjected to a closer scrutiny - especially "The Sisters", "A Little Cloud" and "The Dead". The analytical revision emphasizes not only the "scrupulous meanness" of the style, which is the signature of the sober realism of the stories, but also the symbolic dimension that lies in Joyce's narrative strategies of indirection, in that which is left untold, the absent story (gnomon) that casts its shadow or its light in the typically joycean epiphany, all of which constitutes the poetic and symbolic dimension of Dubliners.

KEY WORDS: James Joyce, Dubliners. 
Publicada en 1914, Dublineses, de James Joyce, es una colección de cuentos sobre vidas malogradas; sobre una ciudad deprimida, estancada; una ciudad que, aletargada, sueña con sus glorias pasadas... Una ciudad que sin embargo no tiene nada que ofrecer a sus habitantes más que mediocridad, sueños de evasión y una subsistencia precaria.

En tres ocasiones Joyce expresa su intención manifiesta con respecto a esta colección de cuentos. En la primera le escribe al editor William Heinemann, a quien en un principio había enviado el manuscrito: "The book is not a collection of tourist impressions but an attempt to represent certain aspects of the life of one of the European capitals" (Ellmann: 109).

En otra le escribe a su hermano Stanislaus: "When you remember that Dublin has been a capital for thousands of years, that it is the 'second' city in the British empire, that it is nearly three times as big as Venice it seems strange that no artist has given it to the world" (111).

Finalmente, a Grant Richards, quien publica Dublineses después de casi diez años de regateos:

My intention was to write a chapter of the moral history of my country and I chose Dublin for the scene because that city seemed to me the centre of paralysis. I have tried to present it to the public under four of its aspects: childhood, adolescence, maturity and public life. The stories are arranged in this order. I have written it for the most part in a style of scrupulous meanness and with the conviction that he is a very bold man who dares to alter in the presentment, still more to deform, whatever he has seen and heard (134).

Uno puede oír claramente el tono defensivo en la primera carta (Dublín no es un lugar exótico que se pueda describir para los turistas, ni su escritura el producto de meras impresiones turísticas). En la segunda hay una clara conciencia y orgullo de la ciudad como una de las grandes capitales de Europa. Ya para la tercera el tono es de resignación y desaliento. Dublín dejó de ser un día una de las grandes capitales europeas para convertirse en el centro de la parálisis de Irlanda. Y si el estilo tiene que ser de escrupulosa mezquindad, aun así, en el estilo se observa el equilibrio de una mirada al mismo tiempo crítica y compasiva. No hay en la representación de estas vidas ni sátira punzante, ni melodrama lacrimoso, simplemente un riguroso realismo cuyo tema primordial es la mezquindad de una ciudad y sus habitantes.

No obstante, en el compás que se abre entre las primeras dos miradas sobre Dublín - la una defensiva, la otra orgullosa - y la tercera, básicamente resignada, se juega por lo menos un siglo de la historia de Irlanda. Con ayuda del historiador J. C. Beckett ("The End of the Irish") haré una breve y muy esquemática revisión de los acontecimientos que van de la Unión a la Gran Hambruna. 


\section{El Acta de la Unión de 1801}

Irlanda fue por siglos colonia de Inglaterra pero conservó siempre una cierta autonomía administrativa, legislativa y financiera, con un parlamento propio que gobernaba desde Dublín. Debido a los problemas económicos del país, pero sobre todo por las consecuencias de la insurrección de 1798, el parlamento de Irlanda firmó, desde 1800, su propia sentencia de muerte.

On 2 August 1800 the Irish parliament met for the last time. However unrepresentative, ineffective or venal, it had stood for more tan five hundred years as a symbol of Ireland's separate place among the nations of Europe (282-283).

En "El Acta de la Unión" de 1801 se especifican varios de los términos: Irlanda habría de unirse a la Gran Bretaña en un solo reino, "el Reino Unido de Gran Bretaña e Irlanda". El parlamento de Dublín sería abolido. Irlanda sería representada en Westminster por cien miembros del parlamento (MP), cuatro Lores Espirituales y veintiocho Lores Temporales, todos anglicanos. La Iglesia anglicana sería reconocida como la iglesia oficial de Irlanda. Irlanda conservaría un ministerio de hacienda (Exchequer) separado, lo cual implicaba hacerse responsable de su propia deuda pública, y bajo la Unión debería cubrir 2/17 de los gastos generales del Reino. Irlanda podía conservar sus propias Cortes de Justicia y su servicio civil. A pesar de que el Primer Ministro de aquella época, William Pitt, y Lord Castlereagh (Robert Stewart, 2nd Marquess of Londonderry) habían prometido que, una vez aprobada la Unión, el paso siguiente sería aprobar la 'Emancipación de los Católicos' (Catholic Emancipation), el compromiso sólo había sido moral y, debido a los problemas de la Gran Bretaña con las guerras napoleónicas, Pitt nunca pudo ejercer influencia sobre el rey ni sobre el Parlamento para que se aprobara esa ley (bill). Así, no se permitiría a los católicos tener puestos públicos bajo la Unión; no habría, en pocas palabras, 'Emancipación de los Católicos' (284 y ss.).

\section{Consecuencias a largo plazo de la Unión de 1801}

Al desaparecer el parlamento los ministros parlamentarios y sus séquitos abandonaron Irlanda, y por tanto vendieron sus casas para poder vivir en Londres. A las grandes mansiones de la aristocracia irlandesa se les dio otro uso; por ejemplo, la del duque de Leinster fue vendida a la Royal Dublin Society; se le construyó otra ala que hasta la fecha funge como la Biblioteca Nacional de Irlanda.

Depresión urbana. Muchas de las casas fueron vendidas y/o abandonadas, sobre todo al norte del río Liffey, pues la mayoría de los nobles y los miembros de la alta burguesía habían seguido en tropel la moda impuesta por el conde de Kildare, más tarde Duke of Leinster, quien construyó su palacio al sur del río. Estas casas al norte 
del río acabaron convirtiéndose en viviendas para la clase media y media baja y, en ocasiones, incluso en vecindades (tenement houses).

Depresión rural. Si el ausentismo fue siempre un flagelo, éste se agravó considerablemente a partir del Acta de la Unión de 1801. Los terratenientes ausentes (absentee landlords) vivían de sus rentas, muchos de ellos en la metrópoli del reino, Londres. ${ }^{1}$ Las rentas no se reinvertían en Irlanda sino que era un capital que salía del país.

El acuerdo de libre comercio entre todos los países de la Unión suponía un alivio a los problemas económicos de Irlanda; a la larga sucedió lo contrario. Las inversiones de capital británico nunca llegaron a Irlanda y, en cambio, cada vez más las tierras se utilizaron como pastura para el ganado que se exportaba a Inglaterra; ahí los ganaderos recibían un mejor precio que de los empobrecidos irlandeses que no tenían para comer carne. Lo mismo ocurría con la cosecha de otros alimentos que eran mejor pagados en Inglaterra. En consecuencia, los campesinos irlandeses - la mitad de la población del país, en su mayoría católicos - se vieron obligados a depender cada vez más de la papa casi como único alimento.

Cuando la Iglesia anglicana se convirtió en la iglesia oficial de todo el reino, los irlandeses, en su mayoría católicos y campesinos pobres, estuvieron obligados, además de pagar renta al Land lord, a pagar el diezmo (tithe) a la Iglesia anglicana, lo cual indignaba, amén de empobrecer más, a los católicos.

A partir de la Unión, Irlanda fue gobernada desde Westminster con un desconocimiento absoluto de los problemas específicos del país. Más aún, el parlamento, preocupado por las guerras contra Napoleón, dedicaba poco tiempo a considerar los problemas de Irlanda - la famosa "Irish Question"; por otra parte, en Westminster se pensaba que los irlandeses siempre exageraban y que, además, se comportaban más como enemigos que como miembros de la Unión.

Los términos de la Unión exigían que Irlanda contribuyera con una parte de los gastos del imperio, lo cual en principio no era necesariamente oneroso, pero lo que nunca se previó fue la prolongación casi indefinida de las guerras napoleónicas, cuyos enormes costos significaron un peso financiero insoportable para Irlanda.

El veto a la Emancipación Católica (Catholic Emancipation) significaba que el status quo de los católicos no se vería jamás beneficiado por la Unión; los católicos seguirían sin tener participación política, puestos públicos o en el parlamento. Esto, claro está, trajo una mayor división entre los anglo-irlandeses protestantes y los irlandeses católicos. Ya desde el siglo XVII esta situación de discriminación a los católicos había propiciado la emigración de muchos irlandeses nobles a países católicos como España y Francia - el fenómeno de los wild geese - y la brutalidad de las guerras de reconquista emprendidas por Oliver Cromwell, aunadas a la masiva confiscación de tierras propiedad de los católicos para dárselas a los colonos ingleses y protestantes.

\footnotetext{
${ }^{1}$ Es inevitable pensar en el paralelismo con México — país que también fue colonia. También los grandes terratenientes y los hacendados vivían de sus rentas en la capital del país o en España.
} 


\section{La "Gran hambruna" (The Great Famine) (1845-1851)}

\section{(Beckett 336 ss)}

A principios de los años cuarenta, Irlanda tenía una población de más de ocho millones de habitantes. La mitad de la población dependía para su subsistencia, de manera casi exclusiva, de la papa. En 1845 se dio la primera infección de la papa ('potato blight'), pero en Londres todos los reportes que venían de Irlanda eran siempre considerados como una exageración.

Difference of opinion about the seriousness of the potato failure did not arise solely from variation in the reports; there was a political reason also. For some years Peel had been moving slowly towards the belief that the corn laws ought to be repealed, and events in Ireland convinced him that he must act at once. For the irreconcilable protectionists, who comprised a great part of Peel's own party, it was, therefore, a matter of policy to minimize the danger of famine. They seized on every favourable report, they declared that the others were exaggerated, and they discouraged the preparation of relief schemes as unnecessary. It was a misfortune for Ireland that the reality of the famine should have become a political question, and that the preparation of remedial measures should have become entangled in one of the bitterest parliamentary conflicts of the nineteenth century (337).

Al año siguiente, 1846, nuevamente, y de manera más devastadora, azota la plaga de la papa, con la consecuente hambruna y la absoluta desesperación de los campesinos. Desde Westminster la única solución que se le dio a la crisis fue ¡venderles granos a los irlandeses!

It is to Peel's credit that he did not allow personal or party interests to interfere with his plans for relief. Early in November 1845 he arranged, on his own responsibility, for the purchase by the government of $£ 100,000$ 's worth of Indian corn in the United States, and for its shipment to Cork. It was not his intention that the government should undertake responsibility for feeding the people; but he believed that by selling this grain cheaply it would be possible to keep down the general price of food, and prevent profiteering. He placed his main reliance, however, on local efforts by the gentry and professional classes; and a relief commission set up by the government in November had, as its first and main task, the organization of local committees, which were to raise funds and distribute food. At the same time the board of Works was to undertake the construction of new roads, the traditional method of providing employment in hard seasons. ${ }^{2}$

Though Peel took the initiative in these measures, their direction fell largely into the hands of Charles Trevelyan... one of the new generation that was transforming the character of British civil service. Trevelyan worked day and night at his task; but his outlook was dominated by the prevailing laissez-faire philosophy, and at times he

\footnotetext{
${ }^{2}$ Habría que recordar una extraña forma de lo que sólo podría llamarse ‘surrealismo irlandés’: los innumerables caminos, construidos durante la Gran Hambruna, ique no llegan a ninguna parte!
} 
gave the impression he was more alarmed lest the Irish should be demoralized by receiving too much help from the government than lest they should die of starvation through not receiving enough (337-338).

Frente a esta situación, la opinión en Inglaterra era que "la pobreza irlandesa debería recibir ayuda de la propiedad irlandesa ("Irish poverty must be supported by irish property") (339). Pero con la hambruna los terratenientes se iban quedando sin rentas. La opinión en Irlanda, en cambio, era que si los irlandeses ayudaban a pagar los costos del imperio como consecuencia de la Unión, que la Unión estaba obligada a corresponder en esta crisis.

Ahora bien, se creía en aquellos tiempos, siguiendo la política del laissez-faire, que era necesario que trabajaran los irlandeses y con lo que ganaran compraran comida. Hubo, por tanto, un reforzamiento de los programas llamados relief Works. La ironía es que en las zonas más golpeadas por la hambruna "the peasant in these areas rarely handled money, and even more rarely used it to purchase food. He paid his rent by his labour; and he and his family lived on the potatoes he grew himself' (340).

Entre 1845 y 1849 la enfermedad se sumo a la hambruna. Fueron enfermedades típicas de la pobreza que diezmaron también a la población: tifo y las llamadas fiebres recurrentes (relapsing fever. Se trata de una infección transmitida por piojos o garrapatas y se caracteriza por episodios repetitivos de fiebre). No es de sorprender que el pánico provocara una emigración masiva.

Emigration, formerly a last desperate remedy, was now the first thing thought of; and there was an almost hysterical rush to leave the country, to escape, at all costs, from the 'doomed and starving island' and find safety elsewhere... from the latter half of 1846 , the panic-stricken crowds were clamorous to be off without delay...

[...] the rate of mortality was high — of emigrants sailing from Liverpool to Canada in 1847, one in fourteen died at sea, of those sailing from Cork, one in nine; and the bitter memory of the 'ships' is firmly entrenched in the folk-tradition of the famine (344-345).

Si en 1841 la población había sido de 8,175,000, para 1851 se había reducido a $6,552,000$. Dos millones habían muerto de hambre, de enfermedad o en tránsito hacia América, en aquellos ‘barcos féretro' (coffin ships). A principios del siglo Xx la población de Irlanda - unos cuatro millones - ya era sólo la mitad de lo que había sido antes de la hambruna. Hoy en día Irlanda aún no se recupera de esa pérdida (población actual: 6.5 millones).

Así, Dublín, fastuosa capital de Europa en el siglo XVIII, con una hermosa arquitectura neoclásica georgiana inspirada, entre otros, en el arquitecto veneciano renacentista Andrea Palladio, y gozando de un cierto auge económico, para principios del siglo XX estaba diezmada y desmoralizada. Vidas malogradas todas. Éste es el centro mismo de la colección de cuentos de Joyce: el relato de vidas malogradas. 


\section{Temas recurrentes en Dublineses}

Varios temas recurren insistentemente a lo largo de muchos de los cuentos:

1. La triada propuesta desde el primer cuento: parálisis (y su contraparte evasión, emigración), gnomon (en tanto que sombra y ausencia) y simonía (la venta de bienes espirituales). Esta triada vuelve una y otra vez en casi todos los cuentos, como lo veremos más tarde.

2. Parálisis, como estancamiento, emblemática en el uso de colores: café, marrón y amarillo; también azul y blanco (virgen María).

3. La frustración de la vida en familia.

4. El siempre acucioso problema del dinero.

5. Alcoholismo.

6. Epifanía y autoconciencia.

7. La propia ciudad como tema.

8. La omnipresencia de la Iglesia católica como una sombra.

9. La cultura y la política en Irlanda.

a) El "Renacimiento Céltico" (Celtic Revival), también llamado Renacimiento literario irlandés. ${ }^{3}$ La revaloración del oeste de Irlanda como la región más auténtica, y del gaélico como la verdadera lengua de Irlanda (Gaelic League).

b) Fanatismo religioso, político y cultural (por ejemplo, Miss Ivors, en "Los muertos", acusa a Gabriel Conroy de ser un "West Briton" porque escribe en un periódico proinglés y prounión). El tema de escandalizar al adversario es constante, insistente y, a la larga, paralizante, pues si un católico, por ejemplo, critica a la Iglesia católica, se considera que le está dando armas al protestante en su contra - está propiciando el escándalo. Y viceversa. Lo mismo ocurre en términos políticos, si un nacionalista critica los movimientos nacionalistas, se cree que le da armas a los unionistas, y viceversa.

Tomemos al azar solamente tres temas, la vida en familia, el alcoholismo y la precariedad de la vida, para abordar los cuentos en panorámica.

Vida en familia. En casi todos los cuentos, la vida en familia es opresiva, sofocante; algunos ejemplos: la frustración de Little Chandler que lo lleva a gritarle a su bebé, en "Una nubecilla"; la brutalidad de Farrington, en "Contrapartes", quien se desquita golpeando al hijo por la disculpa abyecta que le obligaron a pedir en la oficina - sumisión en el trabajo, brutalidad en casa. La violencia del padre de Eveline contra su madre y sus hermanos y ahora contra ella misma.

El alcoholismo. De una u otra manera en casi todos los cuentos hay un alcohólico o por lo menos un borrachín parlanchín, el estereotipo mismo del irlandés. Lenehan, el eterno gorrón de "Dos galanes"; el padre de Eveline — cuyo alcoholismo está impli-

\footnotetext{
${ }^{3}$ Movimiento literario y cultural que quiso rescatar las raíces celtas de la cultura irlandesa, así como un retorno a la lengua originaria de Irlanda, el gaélico. Personajes que se destacan en este movimiento son W. B. Yeats, Lady Gregory, John Millington Synge, George Russell (A. E.), Sean O'Casey, Padraic Colum y Douglas Hyde, entre tantos otros.
} 
cado en los pleitos por el dinero los sábados por la noche-; Jimmy Doyle, el único junior del grupo en "Después de la carrera", atacado de alcoholismo y servilismo, quien por ello pierde hasta el último céntimo de lo que supuestamente iba a invertir con los extranjeros; el carnicero alcohólico con quien se casa Mrs. Mooney, la Madame de "La pensión"; Freddy Malins, en "Los muertos", tan alcohólico que deberá ir a un lugar de rehabilitación. Los borrachos de "Gracia" cuyos pecados se lavan en un retiro ad hoc para empresarios y comerciantes (nuevamente, simonía), y así, muchos más...

La precariedad de la vida. A nadie le alcanza nunca el dinero que gana; la mayoría vive al día, muchos de prestado y otros de gorra. Lenehan, el paradigmático gorrón de Dublineses $y$ del Ulises, deambula por toda la ciudad viendo de dónde consigue algo de dinero y quién le va a pagar el siguiente trago. Farrington, desesperado porque no tiene dinero para un trago, empeña su reloj y recibe una cantidad exorbitante que se gasta completa en la bebida, sin dejar nada para la familia. Eveline tiene eternos pleitos con el padre los significativos sábados en la noche para poder sacarle un poco de dinero para subsistir.

\section{Tres temas omnipresentes en Dublineses: parálisis, gnomon y simonía}

Abordaremos ahora, con más detalle, esto tres grandes temas que aparecen en casi todos los cuentos de Dublineses. Es el primer cuento, "Las hermanas", el que marca la pauta en esta partitura de la frustración y el desaliento. La clave de sol - que en realidad se convierte en la clave de sombra de Dublineses - son tres palabras: parálisis, gnomon y simonía. Ya había dicho Joyce, explícitamente, que para el escenario de sus cuentos escogió Dublín porque le parecía que la ciudad era el centro de la parálisis. Parálisis es una de las primeras palabras que aparece en el cuento; parálisis y su contraparte: el deseo de evasión que constantemente se manifiesta como proyectos de emigración ("Eveline", "Una nubecilla", "Los muertos"). La segunda palabra es gnomon; las dos vertientes de su significado animan temáticamente toda la colección. Por una parte, gnomon es la barra o estilete que proyecta una sombra sobre un reloj de sol para saber la hora; por otro es la sección ausente de un paralelogramo, ausencia que sería su contraparte. En efecto, es ésta una partitura en clave de sombra, proyectada por la opresión de la iglesia y la del fanatismo religioso-político que como fantasmas recorren los cuentos; los excesos del nacionalismo y la grisura de una mediocridad sin fin. Es interesante hacer notar que, en términos de la imaginería de Dublineses, luz y sombra, como la manifestación lírica de gnomon, alternan, contrastan o entran en juegos de claroscuros de manera muy insistente; los cuentos "Las hermanas" y "Araby", en especial, están construidos en torno a incesantes motivos de luz y sombra. Finalmente, la simonía, tema-pecado que extiende sus tentáculos metafóricos a casi todos los habitantes. La simonía es un pecado definido como la compra o venta de lo espiritual por medio de bienes materiales, lo cual incluye cargos eclesiásticos, sacramentos, reliquias, promesas de oración, la gracia, la jurisdicción eclesiástica, la exco- 
munión, etcétera. Este comercio de lo espiritual con lo material se extiende metafóricamente a todas las actividades de los dublineses. Todos se venden o venden sus principios: el gorrón Lenehan vende su elocuencia por un trago, aquí, en "Los dos galanes" y en el Ulises; Gallaher se vende a Inglaterra con todo y corbata (naranja) ("Una nubecilla"); ${ }^{4}$ Mrs. Mooney y Mrs. Kernan venden a sus hijas de distintas maneras ("La pensión“ y "Una madre"); Gabriel Conroy se vende a un periódico pro-británico y por eso Miss Ivors lo ataca diciéndole West Briton ("Los muertos"). Farrington vende su reloj por unos tragos ("Contrapartes"); los borrachos en distintos grados de "Gracia" compran la gracia y el perdón de sus pecados con un retiro donde pueden tratar sus "asuntos" (business) y hacer sus "cuentas" (accounts) con Dios; así, de este modo, la lista se alarga, como diría Macbeth, 'to the crack of Doom'.

En cuanto a la parálisis, es como un sudario que lo cubre todo: tanto la representación del espacio, como la del discurso y la de la acción. Miremos algunos ejemplos de parálisis en conjunción con gnomon, en tanto que segmento ausente, conjunción que se traduce en elipsis, huecos, implicaciones, lo no dicho o dicho a medias; todo aquello que va dibujando una historia ausente, virtual, que se va colando por los huecos de lo no dicho, de las elipsis, de tantas resonancias que nos hablan de otra historia posible.

En el primer cuento, "Las hermanas" — el que pone la pauta_, las elisiones son constantes; todo parece jugarse en una penumbrosa zona de ambivalencia.

(Cotter) "No, I wouldn't say he was exactly... but there was something queer... there was something uncanny about him. I'll tell you my opinion..." (Dubliners: 23).

Old Cotter looked at me for a while. I felt that his little beady black eyes were examining me but I would not satisfy him by looking up from my plate. He returned to his pipe and finally spat rudely into the grate.

"I wouldn't like children of mine," he said, "to have too much to say to a man like that".

"How do you mean, Mr. Cotter?" asked my aunt.

"What I mean is," said old Cotter, "it's bad for children. My idea is: let a young lad run about and play with young lads of his own age and not be... Am I right, Jack?" (1-2).

Las elisiones y lo oblicuo del discurso sugieren algo siniestro que nunca se dice. Ahora bien, si la parálisis del sacerdote es física, la descripción puntual de los síntomas

\footnotetext{
${ }^{4}$ Habría que recordar que el naranja, en contexto irlandés, nunca es simplemente un color sino una postura política. El naranja es el color que eligieron los unionistas, proingleses como distintivo, en memoria de William of Orange, más tarde William III, quien venció al último de los Estuardo católicos que pretendía el trono de Inglaterra y a quien defendían los irlandeses católicos. James Stuart, James II, el último rey católico de Inglaterra, fue depuesto por su yerno William of Orange. Con el apoyo de los irlandeses, James intentó recuperar el trono pero fue derrotado por las huestes naranjas en la emblemática "Battle of the Boyne", el 1 de julio de 1690.
} 
es tal que puede ser leída, según Gifford (29), como "general paralysis of the insane", es decir, paresis: sífilis del sistema nervioso. ${ }^{5}$ De tal modo que demencia, parálisis y los huecos en un discurso ya de por sí oblicuo sugieren implicaciones siniestras, la posibilidad de una historia virtual, diferente de la que estamos leyendo, virtualidad que se adivina en las palabras de Cotter y de las hermanas - "queer, something gone wrong with him". La descripción de la conducta desquiciada de Father Flynn correspondería a los síntomas de la paresis.

"Mind you, I noticed there was something queer coming over him latterly. Whenever I'd bring in his soup to him there I'd find him with his breviary fallen to the floor, lying back in the chair and his mouth open" (30).

"He was too scrupulous always," she said. "The duties of the priesthood was too much for him. And then his life was, you might say, crossed".

"Yes," said my aunt. "He was a disappointed man. You could see that" (31).

"It was that chalice he broke... That was the beginning of it. Of course, they say it was all right, that it contained nothing, I mean. But still... They say it was the boy's fault. But poor James was so nervous, God be merciful to him!” (9-10).

"And was that it?" said my aunt. "I heard something..." Eliza nodded. "That affected his mind," she said. "After that he began to mope by himself, talking to no one and wandering about by himself".

"Wide-awake and laughing-like to himself... So then, of course, when they saw that, that made them think that there was something gone wrong with him..." (10).

La interrelación parálisis/gnomon se subraya aquí de manera insistente: lo no dicho, lo dicho a medias, lo insinuado, la sensación de que hay algo siniestro en el ambiente. Todo lo que resuena en la palabra gnomon - elipsis, huecos, ausencias - informa mucho de la significación del cuento. Como diría Margarita Gasque, "Joyce dice lo que se encubre, encubriendo él mismo lo que dice". ${ }^{7}$ Surgen así innumerables preguntas que podrían perfilar otra historia: ¿cómo y cuándo contrajo la sífilis el padre Flynn?

\footnotetext{
${ }^{5}$ Usually assumed to be the result of the three strokes the priest has suffered, but it may well be the other way around, that the strokes have been caused by the paralysis, since in 1904 the term paralysis was frequently used in medical parlance (and by Joyce) to mean "general paralysis of the insane", i. e. paresis, syphilis of the central nervous system. It is possible to demonstrate that in rewriting the story... Joyce not only added the word "paralysis" but also worked the symptoms of paresis into the boy-narrator's recall of the priest's appearance and manner. Don Gifford, Joyce Annotated. Notes for Dublineses and A Portrait of the Artist as a Young Man. 2a. ed. corregida y aumentada. Berkeley / Los Ángeles / Londres: University of California Press, 1982, p. 29.

${ }^{6} \mathrm{~A}$ menos que se indique otra cosa, las cursivas son mías.

${ }^{7}$ Interesante sugerencia en quiasmo que describe de manera muy sucinta el método joyceano de la indirección. Comunicación oral con la doctora Margarita Gasque, psicoanalista.
} 
¿En qué circunstancias se rompió el cáliz? ¿Por qué se sospecha del niño? ¿Por qué la insistencia de Old Cotter de no dejar a los niños solos con el sacerdote, por mucho que les enseñe?...

Abordemos ahora el cuento "Eveline", para marcar, ahí también, ausencias, implicaciones siniestras, zonas de penumbra que abren el abanico para muchas más lecturas de las que la superficie en apariencia anodina del cuento sugiere. "Eveline" comienza con un nombre siempre evocativo de lo irlandés. Gifford marca la evocación al poema de Tommy Moore "Eveleen's Bower'.

\author{
Oh, weep for the hour \\ When to Eveleen's bow'r \\ The Lord of the valley with false vows came; \\ The moon hid her light \\ From the heavens that night \\ And wept behind the clouds o'er the maiden's shame (Gifford: 49).
}

De manera oblicua se puede establecer un paralelismo implícito entre la falsedad del "Lord of the valley" de la balada de Tommy Moore y la potencial falsedad de Frank, sugiriendo una veta narrativa latente. ¿De veras el marino tiene intenciones de casarse con Eveline y llevársela a Buenos Aires? La referencia a Tommy Moore proyecta la sombra de una historia ausente pero posible —una vez más, gnomon. No obstante, se proyecta también una sombra más siniestra aún, porque el cuento no se llama Eveleen, por muy irlandés que sea el nombre, sino "Eveline". En sus notas a la edición Penguin de 1992, Terence Brown nos remite al monumental estudio de Peter Gay, The Bourgeois Experience, Victoria to Freud: Education of the Senses. En el capítulo VI, "El conocimiento carnal", Gay dice que "[g]ran parte de la pornografía, de hecho, parece un plagio de fantasías infantiles. El predominio de padres que se unen a sus hijas... es un ejemplo. En Eveline, ejemplo victoriano que aparentemente fue revisado más de una vez y que fue muy popular durante décadas, una joven, cuya especialidad es la felación... disfruta de la cópula con sus sirvientes, su hermano y su padre..." (347). ${ }^{8}$

\footnotetext{
${ }^{8}$ Terence Brown sugiere incluso que podría haber ahí una burla velada a George Russell (A. E.): "When it is remembered that this story was written in an earlier form at the suggestion of George Russell for the Irish Homestead ... and that Russell advised Joyce that he should not shock his readership, the possibility arises that the Young author was playing a mischievous joke in using this name and perhaps implying sexual abuse as a subterranean theme" (253-254). De este modo, Joyce escandaliza de manera subterránea a A. E., dando a su cuento el mismo título de la novela pornográfica anónima del siglo XIX: Eveline.

Ahora bien, no me parece tan descabellada esa lectura al recordar también que, aunque alumno modelo, las lecturas de Joyce escandalizaron a varios en esa época, en especial al bibliotecario de Capel Street Lending Library. Su hermano, Stanislaus, en sus memorias, significativamente intituladas My Brother's Keeper, cuenta cómo la censura de Old Grogan, el bibliotecario, tuvo como resultado el que Joyce tuviera que mandar a su hermano a sacar los libros de la biblioteca por él. Un día, al ver la lista, Stanislaus —influido, suponemos, por la mala fama lectora de James - creyó leer, en la lista que llevaba, que la novela de Thomas Hardy, Jude the Obscure, se llamaba ;Jude the Obscene! (Joyce, S.89-90).
} 
Con esta matriz hermenéutica, es muy diferente la lectura del siguiente pasaje: "Even now, though she was over nineteen, she sometimes felt herself in danger of her father's violence. She knew it was that that had given her the palpitations. When they were growing up he had never gone for her like he used to go for Harry and Ernest, because she was a girl but latterly he had begun to threaten her and say what he would do to her only for her dead mother's sake. And now she had nobody to protect her" (30-31).

Una vez más, se perfila otra historia tras la que leemos — gnomon - ¿por qué se siente tan amenazada Eveline, al grado de tener palpitaciones? Esa violencia ¿es sólo física, solamente golpes? O bien, ¿se adivina ahí el abuso sexual como un "tema subterráneo", a decir de Terence Brown? Por otra parte, y siguiendo una vez más a Terence Brown, parece ser que "Going to Buenos Ayres" no remite solamente a la capital de Argentina, cuya bonanza atrajo a tantos migrantes en el siglo XIX y principios del XX, sino que la frase en esa época era una frase coloquial (slang) que significaba 'darse a la prostitución'. ${ }^{9}$ Por eso es patética la fantasía que construye Eveline en torno a su vida futura en Buenos Aires: "she was about to explore another life with Frank. Frank was very kind, manly, open-hearted. She was to go away with him by the night-boat to be his wife and to live with him in Buenos Ayres where he had a home waiting for her" (31).

Otro aspecto ambiguo del relato es la circularidad y la patente inmovilidad: principio, medio y fin están marcados por la pasividad: "She sat at the window watching the evening invade the avenue. Her head was leaned against the window curtains and in her nostrils was the odour of dusty cretonne. She was tired" (29).

Finalmente, el gran enigma del cuento es el estatuto narrativo y ontológico de Frank. Por una parte, la referencia a Buenos Aires, como hemos visto, abre una zona de ambigüedad muy grande; por otra parte, la caracterización de Frank es en sí misma sospechosa, por ser demasiado libresca.

He was awfully fond of music and sang a little. People knew that they were courting and, when he sang about the lass that loves a sailor, she always felt pleasantly confused. He used to call her Poppens out of fun. First of all it had been an excitement for her to have a fellow and then she had begun to like him. He had tales of distant countries. He had started as a deck boy at a pound a month on a ship of the Allan Line going out to Canada. He told her the names of the ships he had been on and the names of the different services. He had sailed through the Straits of Magellan and he told her stories of the terrible Patagonians.

He had fallen on his feet in Buenos Ayres, he said, and had come over to the old country just for a holiday. Of course, her father had found out the affair and had forbidden her to have anything to say to him.

"I know these sailor chaps," he said.

\footnotetext{
${ }^{9}$ Buenos Ayres. Capital of Argentina, in the nineteenth century and early twentieth century a thriving and wealthy city which attracted many European immigrants and adventurers. The phrase 'Going to Buenos Ayres' was also slang for taking up a life of prostitution (254-255).
} 
One day he had quarrelled with Frank and after that she had to meet her lover secretly.

The evening deepened in the avenue. [...]

Her time was running out but she continued to sit by the window (32).

¿Cómo habría que leer todo esto? Hay varias posibilidades:

1. Puede uno leer el final literalmente como otro ejemplo de parálisis: Eveline no se atreve a dejar el infierno en que vive.

2. Puede uno dudar de Frank, quien le cuenta a Eveline 'talltales' de marinos para seducirla y luego abandonarla; nunca pensó llevársela a Buenos Aires (o sí, ¿con intenciones de proxeneta?).

3. Finalmente, se podría pensar que es solamente a partir de las románticas historias de Frank que Eveline se hace la fantasía de irse con él, pero es sólo eso: fantasía. En tal caso, lo terrible es que ni en la fantasía se atreve a romper con la parálisis y la opresión de su vida familiar.

De cualquier manera, la pregunta que se impone es: ¿por qué se abre tanto el abanico hermenéutico en una historia aparentemente tan sencilla? El meollo del asunto está en un hueco — gnomon, nuevamente-, la inmensa elipsis en el penúltimo párrafo. A lo largo de todo el cuento Eveline ha estado sentada junto a la ventana, pasivamente; su mente viaja, de manera alternada, hacia el pasado y hacia el futuro, pero no se mueve de la ventana. No es sino hasta después del recuerdo de la demencia pseudogaélica de la madre que se levanta, pero se levanta para saltar al vacío de una elipsis inexplicable. Considerando lo puntual, lo escrupuloso en la representación de la triste vida de Eveline, salta a la vista este hueco que no dice nada de cómo pasa esta muchacha de la inactividad — reiteradamente marcada — al bullicio de un muelle, para finalmente revertir el movimiento hacia una pasividad animal. Pasividad al inicio, pasividad en medio, pasividad al final, como si nunca se hubiera movido de la ventana... Parálisis.

El salto al abismo de la elipsis es de tal magnitud que llegamos a dudar incluso de la realidad de esa última escena: ya no sabemos si en verdad salió de su casa y fue al muelle, si todo el fracaso está en su imaginación; si Frank le quería tomar el pelo, o si, incluso, Frank existe, dada su constitución sospechosamente libresca.

\section{La epifanía y la escrupulosa mezquindad de la escritura de Dublineses}

Este estilo de escrupulosa mezquindad es aquel con el que Joyce retrata Dublín y a los dublineses de manera rigurosamente realista, casi podríamos decir puntillosamente realista. Habría que recordar que casi todos los cuentos los escribió ya en el exilio. Sabemos de la obsesión casi fetichista por la precisión del detalle, por eso su hermano Stanislaus, y junto con él todos sus amigos, sufrían el acoso constante de Joyce para que le mandaran vestigios materiales de lo real — boletos de tranvía, programas de conciertos, reclamos publicitarios, resultados de las carreras de automóviles, etcétera-; el 
mismo Joyce trabajaba sobre un mapa de Dublín para ser preciso en los pormenores del deambular de sus personajes. Mismo método en la construcción del Ulises.

Y sin embargo, su realismo no es meramente naturalista sino que está cribado por el simbolismo del juego de luz y sombra, del juego entre presencia y ausencia, y de lo dicho y lo no dicho, como hemos observado; en una palabra, un estilo de escrupulosa mezquindad atravesado por un estilo de indirección que abre enigmas más allá de todo realismo. Finalmente, los cuentos están tamizados por la luz de las epifanías —otra manera de decir lo no dicho. Sabemos que la epifanía marca la revelación que tienen los Reyes Magos de la divinidad de Cristo en medio de la más absoluta pobreza — la del emblemático pesebre. Metafóricamente hablando, para Joyce es la revelación de una verdad sobre el personaje, sobre un episodio, una situación, un objeto; en suma, la manifestación del sentido ausente en condiciones que en ocasiones rayan en la sordidez. En Stephen Hero, Joyce escribe:

By an epiphany he meant a sudden spiritual manifestation, whether in the vulgarity of speech or gesture or in a memorable phrase of the mind itself ... He believed that it was for the man of letters to record these epiphanies with extreme care, seeing that they themselves are the most delicate and evanescent moments (216).

The mind considers the object in whole and in part, in relation to itself and to other objects... So the mind receives the impression of the symmetry of the object. The mind recognises that the object is in the strict sense of the word, a thing, a definitely constituted entity... For a long time I couldn't make out what Aquinas meant. He uses a figurative word (a very unusual thing for him) but I have solved it. Claritas is quidditas. After the analysis which discovers the second quality the mind makes the only logical possible synthesis and discovers the third quality. This is the moment which I call epiphany. First we recognise that the object is one integral thing, then we recognise that it is an organized composite structure, a thing in fact: finally, when the relation of the parts is exquisite, when the parts are adjusted to the special point, we recognize that it is that thing which it is. Its soul, its whatness, leaps to us from the vestment of its appearance. The soul of the commonest object, the structure of which is adjusted, seems to us radiant. The object achieves its epiphany (218). ${ }^{10}$

Algo en lo que sería necesario insistir es que la epifanía no necesariamente conlleva éxtasis o intensa felicidad; lo que subraya es la súbita manifestación espiritual de algo, incluso vulgar, vergonzoso o sórdido. Quizá una de las epifanías joyceanas más notables por la sordidez que deviene lirismo es el momento, en el capítulo v de $E l$ retrato del artista adolescente, en el que Stephen Dedalus descubre un piojo que baja por su cuello:

She had passed through the dusk. And therefore the air was silent save for one soft hiss that fell. And therefore the tongues about him had ceased their babble. Darkness was falling.

\footnotetext{
${ }^{10}$ Cursivas en el original. Las negritas son mías.
} 
Darkness falls from the air.

A trembling joy, lambent as a faint light, played like a fairy host around him. But why? Her passage through the darkening air or the verse with its black vowels and its opening sound, rich and lute like?...

A louse crawled over the nape of his neck and, putting his thumb and forefinger deftly beneath his loose collar, he caught it. He rolled its body, tender yet brittle as a grain of rice, between thumb and finger for an instant before he let it fall from him and wondered would it live or die. There came to his mind a curious phrase from Cornelius a Lapide which said that the lice born of human sweat were not created by God with the other animals on the sixth day. But the tickling of the skin of his neck made his mind raw and red. The life of his body, ill clad, ill fed, louse-eaten, made him close his eyelids in a sudden spasm of despair and in the darknesshe saw the brittle bright bodies of lice falling from the air and turning often as they fell. Yes, and it was not darkness that fell from the air. It was brightness.

\section{Brightness falls from the air.}

He had not even remembered rightly Nash's line. All the images it had awakened in him were false. His mind bred vermin. His thoughts were lice born of the sweat and sloth (A Portrait: 205-207). ${ }^{11}$

En este texto memorable del Retrato la epifanía surge como una súbita manifestación espiritual a partir de un complejo proceso mental: el recuerdo falso de un verso del poeta isabelino Thomas Nash que lo lleva a una serie de lucubraciones que después Stephen mismo apreciará como falsas. La aparición del piojo en tanto objeto de contemplación (quidditas) se entreteje con la reverberación del verso de Nashy en ese momento surge la doble revelación que lo lleva, por un lado, a recordar correctamente el verso, que habla no de oscuridad sino de claridad (claritas) — "Brightness falls from the air" - , y por otro, le provoca un 'spasm of despair', porque "His mind bred vermin. His thoughts were lice born of the sweat and sloth".

Ahora bien, en Dublineses la transformación espiritual surgida de una revelación está simbolizada a veces en los muchos juegos de luz y oscuridad: por ejemplo, en el cuerpo y ropa de la hermana de Mangan en "Araby", o en el mismo juego de luz y sombra en la falda de Gretta en "Los muertos".

Gabriel had not gone to the door with the others. He was in a dark part of the hall gazing up the staircase. A woman was standing near the top of the first flight, in the shadow also. He could not see her face but he could see the terracotta and salmonpink panels of her skirt which the shadow made appear black and white. It was his wife. She was leaning on the banisters, listening to something. Gabriel was surprised at her stillness and strained his ear to listen also. [...]

\footnotetext{
${ }^{11}$ Versos citados en cursivas en el original.
} 
He stood still in the gloom of the hall, trying to catch the air that the voice was singing and gazing up at his wife. There was grace and mystery in her attitude as if she were a symbol of something. He asked himself what is a woman standing on the stairs in the shadow, listening to distant music, a symbol of. If he were a painter he would paint her in that attitude. Her blue felt hat would show off the bronze of her hair against the darkness and the dark panels of her skirt would show off the light ones. Distant Music he would call the picture if he were a painter (210-211).

El juego de luz y sombra es un motivo constante en Joyce. Esta descripción de la falda de Gretta, y la mujer misma, parcialmente iluminada, evoca otra muy similar en el cuento "Araby", al describir a la hermana de Mangan:

She was waiting for us, her figure defined by the light from the half-opened door (22).

Her brother and two other boys were fighting for their caps and I was alone at the railings. She held one of the spikes, bowing her head towards me. The light from the lamp opposite our door caught the white curve of her neck, lit up her hair that rested there and, falling, lit up the hand upon the railing. It fell over one side of her dress and caught the white border of a petticoat, just visible as she stood at ease (24).

En ambos casos, la mujer se convierte en un objeto de contemplación focalizado metonímicamente en el juego de luz y sombra sobre su atuendo. Habría que recordar en este momento lo dicho por Joyce: "when the relation of the parts is exquisite, when the parts are adjusted to the special point, we recognize that it is that thing which it is. Its soul, its whatness, leaps to us from the vestment of its appearance. The soul of the commonest object, the structure of which is adjusted, seems to us radiant. The object achieves its epiphany". Tanto en "Araby" como en "Los muertos", la mujer está literalmente iluminada; la contemplación de la amada la convierte en un objeto que irradia luz, que incluso se transfigura, como en el caso de Gretta Conroy, en un símbolo, en un objeto de arte. Es significativo, además, que en los dos cuentos el acto de la contemplación guarda un mismo arreglo espacial: el contemplador abajo, al pie de la escalera; la amada, transfigurada por el juego de luz y sombra, arriba.

Ahora bien, no se puede decir que la epifanía en Dublineses sea algo inmediatamente detectable, ni única — una por cuento - ni para quién es, ¿el personaje o el lector? Una vez más, en "Araby" hay una terrible contigüidad entre lo sagrado del amor recién descubierto y la mezquindad de la ciudad:

Her image accompanied me even in places the most hostile to romance. On Saturday evenings when my aunt went marketing I had to go to carry some of the parcels. We walked through the flaring streets, jostled by drunken men and bargaining women, amid the curses of labourers, the shrill litanies of shop-boys who stood on guard by the barrels of pigs' cheeks, the nasal chanting of street-singers, who sang a come-allyou about O'Donovan Rossa, or a ballad about the troubles in our native land. These 
noises converged in a single sensation of life for me: I imagined that I bore my chalice safely through a throng of foes $(22-23) .{ }^{12}$

O bien la epifanía se da también al final del cuento; cuál es la naturaleza de la epifanía no siempre es del todo claro. Nuevamente, en "Araby", el cuento termina con una dolorosa revelación: "Gazing up into the darkness I saw myself as a creature driven and derided by vanity; and my eyes burned with anguish and anger" (28).

¿Surge la epifanía del diálogo vulgar que escucha el niño en el bazar ya casi oscuro? ¿Dónde está la vanidad: en estar enamorado y haber querido comprarle algo a la amada? ¿La epifanía es la revelación de la mediocridad de su vida, con un tío borracho que olvidó que iba a ir al bazar y nunca llegó a tiempo para darle dinero?

De la misma manera ¿cuál es la epifanía de Little Chandler en "Una nubecilla”? ¿La escrupulosa mediocridad en la que vive, proyectada sobre una ciudad que de manera prosopopéyica le devuelve su propia imagen: una vida atrofiada, raquítica (a stunted life)? Porque es él quien se mira, a pesar suyo, en el espejo de las pobres casas atrofiadas:

Little Chandler quickened his pace. For the first time in his life he felt himself superior to the people he passed. For the first time his soul revolted against the dull inelegance of Capel Street. There was no doubt about it: if you wanted to succeed you had to go away. You could do nothing in Dublin. As he crossed Grattan Bridge he looked down the river towards the lower quays and pitied the poor stunted houses. They seemed to him a band of tramps, huddled together along the river-banks, their old coats covered with dust and soot, stupefied by the panorama of sunset and waiting for the first chill of night to bid them arise, shake themselves and begone. He wondered whether he could write a poem to express his idea. Perhaps Gallaher might be able to get it into some London paper for him. Could he write something original? He was not sure what idea he wished to express but the thought that a poetic moment had touched him took life within him like an infant hope. He stepped onward bravely (68).

Como en el caso de Gabriel Conroy, la contemplación de un objeto lo lleva a sentirse artista y a desear plasmar esa visión; Gabriel, en un cuadro; Little Chandler, en un poema. ¿Es ésa la epifanía? ¿Se podría hablar de epifanías en serie hasta llegar a la final? Porque es indudable que hay una importante epifanía al final de "Una nubecilla". Después de gritarle a su bebé y lanzarlo a un paroxismo de llanto, ¿de qué se arrepiente Little Chandler?

A dull resentment against his life awoke within him. Could he not escape from his little house? Was it too late for him to try to live bravely like Gallaher? Could he go to London? There was the furniture still to be paid for. If he could only write a book and get it published, that might open the way for him. [...]

\footnotetext{
${ }^{12}$ Cursivas en el original. Las negritas son mías.
} 
Little Chandler felt his cheeks suffused with shame and he stood back out of the lamplight. He listened while the paroxysm of the child's sobbing grew less and less; and tears of remorse started to his eyes (80-81).

La enorme ambigüedad de esta epifanía es evidente. ¿De qué se arrepiente Little Chandler?, ¿de su propia violencia contra el bebé, de su vanidad, de su servilismo, de su matrimonio, de su vida entera? ¿Para quién es la epifanía?, ¿para él, para nosotros, para los dos?

\section{Más allá de la escrupulosa mezquindad de la escritura realista: el lirismo de la epifanía en "Los muertos"}

Destaca entre todos los cuentos de Dublineses, el último, "Los muertos", no sólo por su extensión sino por su tono. A diferencia de otros cuentos, aquí no priva la mezquindad — si bien a la larga los personajes nunca logran escapar del tinte de la mediocridad - sino la hospitalidad y generosidad de las hermanas Morkan. ${ }^{13}$ El baile, ya sea en primer plano o en el fondo, le confiere esta tonalidad festiva al relato. Pero sobre todo la cena descrita con gran lujo de detalles y prodigalidad cambia la grisura y precariedad de la mayoría de los otros cuentos en una fiesta de color y de abundancia. Es el grado de iconicidad en la descripción de las viandas que adornan la mesa lo que marca la pauta de abundancia, si bien en cierto sentido aparente:

A fat brown goose lay at one end of the table and at the other end, on a bed of creased paper strewn with sprigs of parsley, lay a great ham, stripped of its outer skin and peppered over with crust crumbs, a neat paper frill round its shin and beside this was a round of spiced beef. Between these rival ends ran parallel lines of side-dishes: two little minsters of jelly, red and yellow; a shallow dish full of blocks of blancmange and red jam, a large green leaf-shaped dish with a stalk-shaped handle, on which lay bunches of purple raisins and peeled almonds, a companion dish on which lay a solid rectangle of Smyrna figs, a dish of custard topped with grated nutmeg, a small bowl full of chocolates and sweets wrapped in gold and silver papers and a glass vase in which stood some tall celery stalks. In the centre of the table there stood, as sentries to a fruit-stand which upheld a pyramid of oranges and American apples, two squat old-fashioned decanters of cut glass, one containing port and the other dark sherry. On the closed square piano a pudding in a huge yellow dish lay in waiting and behind it were three squads of bottles of stout and ale and minerals, drawn up according to the colours of their uniforms, the first two black, with brown and red labels, the third and smallest squad white, with transverse green sashes.

Gabriel took his seat boldly at the head of the table and, having looked to the edge of the carver, plunged his fork firmly into the goose. He felt quite at ease now for

\footnotetext{
${ }^{13}$ Interesante simetría: dos hermanas en el primer cuento; dos, en el último.
} 
he was anexpert carver and liked nothing better than to find himself at the head of a well-laden table (197).

El espectáculo exterior, si bien espléndido y estimulante para los sentidos y para la sociabilidad, encubre sin embargo las preocupaciones mezquinas y egocéntricas de Gabriel: que si su discurso será comprendido, que si tal vez no debiera citar a tal poeta... Se preocupa también de cómo va a recibir su discurso Miss Ivors con quien está profundamente resentido por haberlo llamado "West Briton". En fin todas estas pequeñas preocupaciones quedarán enterradas bajo la hermosa nieve de la serie de epifanías al final del cuento.

Quizá la epifanía más compleja (o más bien una serie de epifanías) está aquí, en "Los muertos", y esto es también lo que distingue a este cuento de los demás. Hemos visto ya la epifanía que inicia la serie, cómo Gretta Conroy es descrita básicamente en términos plásticos: de pie en el rellano de la escalera que en conjunción con la sombra le forman un marco (gnomon, nuevamente); el juego de los claroscuros sobre su falda hace que resalte el cobrizo de su pelo, y los cuadros oscuros de su falda hacen resaltar más los claros. El efecto estetizante de la descripción es evidente y es ya una forma de transfiguración del objeto descrito; es lo que provoca la súbita manifestación espiritual en el alma de Gabriel: un deseo de fijar en un cuadro ideal esa imagen de luz y oscuridad.

Al llegar al hotel, en un impulso vagamente romántico, Gabriel le pide al portero que se lleve la vela: "A ghostly [ghastly] light from the street lamp lay in a long shaft from one window to the door.$^{14}$ Gabriel threw his overcoat and hat on a couch and crossed the room towards the window. He looked down into the street in order that his emotion might calm a little. Then he turned and leaned against a chest of drawers with his back to the light. [...] She turned away from the mirror slowly and walked along the shaft of light towards him" (217-218).

El juego de claroscuros aquí forma una figura interesante: un haz de luz atravesando la oscuridad. Han sido tan insistentes las imágenes de luz-oscuridad no sólo en éste sino en varios de los cuentos de la colección — los dos primeros en especial- que la imagen del haz de luz adquiere una carga simbólica importante. Inmediatamente viene a la imaginación la naturaleza invertida de la figura con respecto a la implicada en la palabra gnomon: si una de las acepciones de gnomon es el estilete de sombra que se proyecta sobre un cuadrante solar; aquí un haz de luz se proyecta sobre 'el cuadrante oscuro' de una habitación de hotel. La luz es calificada de fantasmal (ghostly) en alguna ediciones, de cadavérica (ghastly) en otras. En todo caso, ambos calificativos marcan la pauta para el desarrollo del tema final que es el de la conciencia de la mortalidad. Más aún, considerando que la descripción del haz de luz está focalizada en Gabriel, el calificativo ghastly/ghostly sugiere también su actitud frente a luz, a la cual, significa-

\footnotetext{
${ }^{14}$ Es interesante hacer notar que en otras ediciones la palabra no es ghostly sino ghastly. De hecho en la edición de 1914, la de Grant Richards, la palabra es, significativamente, ghastly.
} 
tivamente, le da la espalda. Por contraste, y de manera simbólica, Gretta llega a él por el camino de la luz.

Más tarde, después de la crisis que sufre Gretta al recordar cómo Michael Furey murió por ella, las epifanías en cascada se desatan una tras otra en el alma de Gabriel:

She was fast asleep.

Gabriel, leaning on his elbow, looked for a few moment un resent fully on her tangled hair and half-open mouth, listening to her deep-drawn breath. So she had had that romance in her life: a man had died for her sake. It hardly pained him now to think how poor a part he, her husband, had played in her life. He watched her while she slept, as though he and she had never lived together as man and wife.

His curious eyes rested long upon her face and on her hair: and, as he thought of what she must have been then, in that time of her first girlish beauty, a strange, friendly pity for her entered his soul. He did not like to say even to himself that her face was no longer beautiful, but he knew that it was no longer the face for which Michael Furey had braved death.

Perhaps she had not told him all the story. His eyes moved to the chair over which she had thrown some of her clothes. A petticoat string dangled to the floor. One boot stood upright, its limp upper fallen down: the fellow of it lay upon its side. He wondered at his riot of emotions of an hour before. From what had it proceeded? From his aunt's supper, from his own foolish speech, from the wine and dancing, the merrymaking when saying good-night in the hall, the pleasure of the walk along the river in the snow. Poor Aunt Julia! She, too, would soon be a shade with the shade of Patrick Morkan and his horse. He had caught that haggard look upon her face for a moment when she was singing Arrayed for the Bridal. Soon, perhaps, he would be sitting in that same drawing-room, dressed in black, his silk hat on his knees. The blinds would be drawn down and Aunt Kate would be sitting be side him, crying and blowing her nose and telling him how Julia had died. He would cast about in his mind for some words that might console her, and would find only lame and useless ones. Yes, yes: that would happen very soon.

The air of the room chilled his shoulders. He stretched himself cautiously along under the sheets and lay down beside his wife. One by one, they were all becoming shades. Better pass boldly into that other world, in the full glory of some passion, than fade and wither dismally with age. He thought of how she who lay beside him had locked in her heart for so many years that image of her lover's eyes when he had told her that he did not wish to live.

Generous tears filled Gabriel's eyes. He had never felt like that himself towards any woman, but he knew that such a feeling must be love. The tears gathered more thickly in his eyes and in the partial darkness he imagined he saw the form of a young man standing under a dripping tree. Other forms were near. His soul had approached that region where dwell the vast hosts of the dead. He was conscious of, but could not apprehend, their wayward and flickering existence. His own identity was fading out into a grey impalpable world: the solid world itself, which these dead had one time reared and lived in, was dissolving and dwindling. A few light taps upon the pane made him turn to the window. It had begun to snow again. He watched sleepily the flakes, silver and dark, falling obliquely against the lamplight. The time had come for him to set out on his journey westward. Yes, the newspapers were right: snow was 
general all over Ireland. It was falling on every part of the dark central plain, on the treeless hills, falling softly upon the Bog of Allen and, farther westward, softly falling into the dark mutinous Shannon waves. It was falling, too, upon every part of the lonely churchyard on the hill where Michael Furey lay buried. It lay thickly drifted on the crooked crosses and headstones, on the spears of the little gate, on the barren thorns. His soul swooned slowly as he heard the snow falling faintly through the universe and faintly falling, like the descent of their last end, upon all the living and the dead (223-225).

La serie se inicia con la contemplación de su mujer dormida, y es como si el sueño de Gretta lo llevara en el tiempo a contemplar a aquella joven que había inspirado un amor tan profundo en Michael Furey; al contemplarla se da en él una transformación espiritual: el hombre más o menos fatuo y lleno de sí mismo que hemos visto a lo largo del cuento, preocupado por el efecto que pueda tener su discurso, despreciando más que menos la capacidad intelectual de su público y añorando un impacto en su rival ausente, Miss Ivors, ese hombre sale de su ego, por primera vez, para sentir con y por el otro: "a strange, friendly pity for her entered his soul". Más aún, su piedad se irradia a otros y le abre la conciencia de la mortalidad; es entonces que se da cuenta que la vida de la tía Julia está llegando a su fin: "Soon, perhaps, he would be sitting in that same drawing-room, dressed in black, his silk hat on his knees". Y esa conciencia de la mortalidad le cambia el sentido a su vida; amor y muerte se funden y confunden en una revelación: "Better pass boldly into that other world, in the full glory of some passion, than fade and wither dismally with age". El momento de la revelación coincide con una manifestación física que está presente en algunos de los personajes de los otros cuentos: las lágrimas. En el niño de "Araby" son lágrimas de angustia y rabia: "Gazing up into the darkness I saw myself as a creature driven and derided by vanity; and my eyes burned with anguish and anger." Las de Little Chandler son de arrepentimiento: "tears of remorse started to his eyes". Significativamente, las de Gabriel son lágrimas generosas, lágrimas que rompen el cerco del ego para acercarse al otro, para concebirlo en su imaginación: "The tears gathered more thickly in his eyes and in the partial darkness he imagined he saw the form of a young man standing under a dripping tree". Luego, el fantasma de Michael Furey se multiplica: gracias al lirismo de la prosa, Gabriel, cayendo en un estado de duermevela, emprende una suerte de mítico descenso al infierno: "Other forms were near. His soul had approached that region where dwell the vast hosts of the dead. He was conscious of, but could not apprehend, their wayward and flickering existence. His own identity was fading out into a grey impalpable world: the solid world itself, which these dead had one time reared and lived in, was dissolving and dwindling". Las huestes de los muertos tienen otra historia que contar, no una sobre la mezquindad y la mediocridad que hasta hoy siente Gabriel que ha sido la historia de su vida, sino un impulso renovador que todo lo permea. De manera paradójica, ese impulso renovador le sugiere partir hacia el oeste: "The time had come for him to set out on his journey westward". La sola dirección abre un abanico de sentidos, si bien en apariencia contradictorios, muy sugerentes en distintos 
niveles de significación. El más inmediato es el mítico; este descenso al Hades se da geográficamente, como habrá de darse en el Ulysses, en términos de un desplazamiento hacia el (nor)oeste, dirección implicada en la situación mítico-geográfica del Hades homérico. Así, la conciencia abierta a la mortalidad se dirige naturalmente hacia el oeste, el lugar de la muerte; Michael Furey, la tía Julia, el tío Patrick Morkan, junto con su caballo, él mismo tal vez, todos muertos o cerca de la muerte. Por otra parte, en la ríspida discusión que tiene Gabriel con Miss Ivors, figura la insistencia fanática de que lo auténtico en Irlanda es su lengua originaria, el gaélico, y su tradición cultural, conservada de manera más pura en el oeste de Irlanda. El vago deseo de Gabriel por irse al oeste ¿implica admitir que un regreso al origen sería necesario?, ¿que su orientación espiritual hacia Europa (al este) ha estado equivocada y que es hora de rectificar?; ¿que habiendo vivido una vida tan mediocre es hora de morir simbólicamente, de transfigurarse? Lo cierto es que en este ambiguo final se da otra transfiguración, tanto en el nivel de la prosa como en el de la historia. Tal como estaba anunciada en esa especie de gnomon invertido (la figura del haz de luz que cruza la oscuridad de la habitación) las sombras en torno a Gabriel acaban transformándose en tantos copos de nieve que van cubriendo la tierra: de la sombra a la luz, de la oscuridad a la blancura impoluta de la nieve. En tanto, una prosa poética arrulla con sus ritmos el final del cuento. Hemos pasado así de la escrupulosa mezquindad de la escritura al lirismo de una prosa poética que ya anuncia la de El retrato del artista adolescente. Destacan, de manera muy especial, los efectos rítmicos que producen las aliteraciones y los quiasmos que salpican el último párrafo: falling softly... / softly falling; falling faintly... / faintly falling; His soul swooned slowly.

La resonancia con uno de los pasajes memorables del Retrato, al final del capítulo IV, es especialmente significativa, pues se trata ahí, también, de una de las grandes epifanías de la novela:

A girl stood before him in midstream, alone and still, gazing out to sea. She seemed like one whom magic had changed into the likeness of a strange and beautiful seabird. Her long slender bare legs were delicate as a crane's and pure save where an emerald trail of seaweed had fashioned itself as a sign upon the flesh. Her thighs, fuller and soft-hued as ivory, were bared almost to the hips, where the white fringes of her drawers were like feathering of soft white down. Her slate-blue skirts were kilted boldly about her waist and dovetailed behind her. Her bosom was as a bird's, soft and slight, slight and soft as the breast of some dark-plumaged dove. But her long fair hair was girlish: and girlish, and touched with the wonder of mortal beauty, her face.

She was alone and still, gazing out to sea [...] a faint flame trembled on her cheek.

- Heavenly God! cried Stephen's soul, in an outburst of profane joy.

He turned away from her suddenly and set off across the strand. His cheeks were aflame; his body was aglow; his limbs were trembling. On and on and on and on he strode, far out over the sands, singing wildly to the sea, crying to greet the advent of the life that had cried to him.

Her image had passed into his soul forever and no word had broken the holy silence of his ecstasy (A Portrait: 150). 
Este pasaje está animado no solamente por los ritmos generados por quiasmos y aliteraciones, sino por la transfiguración poética de la muchacha en pájaro, un ser alado, el único digno de acompañar simbólicamente al incipiente poeta en su vuelo: Ícaro deseoso de transmutar en el crisol de su alma la conciencia informe de su raza en un objeto precioso, radiante. Al contemplar a esta muchacha Stephen la desviste de su apariencia y descubre su luz interior, parafraseando a Joyce, su alma salta hasta nosotros desvestida de su apariencia. El alma del objeto más humilde, cuya estructura está ajustada de esta manera, nos parece radiante. El objeto logra su epifanía. Y, de este modo, el poeta desviste también el estilo de su apariencia mezquina para revelárnoslo como un objeto radiante de luz. Es ésta, como en "Los muertos", una revelación fulgurante: la epifanía del estilo.

\section{Obras citadas}

Beckett, J. C. "From the Union to Catholic Emancipation". The Making of Modern Ireland, 1603-1923. Londres: Faber \& Faber, 1966. Impreso.

. "The End of the Irish Parliament". The Making of Modern Ireland, 16031923. Londres: Faber \& Faber, 1966. Impreso.

Ellmann, Richard. Letters of James Joyce. Vol. II. Nueva York: Viking Press, 1966. Impreso.

GAY, Peter. La experiencia burguesa. De Victoria a Freud. I La educación de los sentidos. Trad. Evangelina NiÑo DE LA SELVA. México: FCE, 1992. Impreso.

GIfFord, Don. Joyce Annotated. Notes for Dubliners and A Portrait of the Artist as a Young Man. 2a. ed. revisada y aumentada. Berkeley: University of California Press, 1982. Impreso.

Joyce, James. A Portrait of the Artist as a Young Man. Ed. John Paul RiQuELme. A Norton Critical Edition. Nueva York: W. W. Norton \& Company, 2007. Impreso.

. Dubliners. Introd., apénd. y notas Terence BROwN. Londres: Penguin Books, 1992. Impreso.

. Stephen Hero. Londres: Jonathan Cape, 1969 [1944]. Impreso.

Joyce, Stanislaus. My Brother's Keeper. Introd. Richard Ellmann. Pref. T. S. ELIOT. Londres: Faber \& Faber, 1958. Impreso. 
